OPEN

SUBJECT AREAS:

CONDENSED-MATTER

PHYSICS

FLUIDS

MATERIALS SCIENCE

PHYSICS

Received

16 August 2012

Accepted

11 October 2012

Published

14 November 2012

Correspondence and requests for materials should be addressed to

J.C. ljohan.

christensen@gmail.

com)

\section{Tunable acoustic double negativity metamaterial}

\author{
Z. Liang ', M. Willatzen², J. Li' \& J. Christensen ${ }^{3}$
}

${ }^{1}$ Department of Physics and Materials Science, City University of Hong Kong, Tat Chee Avenue, Kowloon Tong, Hong Kong, ${ }^{2}$ Mads Clausen Institute, University of Southern Denmark, Alsion 2, DK-6400 Sønderborg, Denmark, ${ }^{3}$ IQFR - CSIC Serrano 119, 28006 Madrid, Spain.

Man-made composite materials called "metamaterials" allow for the creation of unusual wave propagation behavior. Acoustic and elastic metamaterials in particular, can pave the way for the full control of sound in realizing cloaks of invisibility, perfect lenses and much more. In this work we design acousto-elastic surface modes that are similar to surface plasmons in metals and on highly conducting surfaces perforated by holes. We combine a structure hosting these modes together with a gap material supporting negative modulus and collectively producing negative dispersion. By analytical techniques and full-wave simulations we attribute the observed behavior to the mass density and bulk modulus being simultaneously negative.

( lassical waves such as sound and light have recently been put to the test in the challenges for cloaking objects $^{1-8}$ and realizing negative refraction ${ }^{9-18}$. Those concepts are just a few of recent fascinating phenomena which are consequences of artificial electromagnetic (EM) or acoustic metamaterial designs. Perfect imaging ${ }^{19}$ or enhanced transmission of waves in subwavelength apertures ${ }^{20}$ are other disciplines within the scope of metamaterials which have received considerable attention both from a theoretical and experimental point of view ${ }^{21-24}$. Smith et al. designed an EM composite material in which the electric and magnetic response act simultaneously to exhibit a negative effective index of refraction band ${ }^{25}$. The idea was to tune the resonance of a periodic array of split ring resonators such that the negative permeability would occur below the plasma frequency of a metallic rodded medium where the electric response is negative ${ }^{26,27}$. An advanced version of this design is provided by the so-called fishnet structure. In its various build-ups it contains a high figure-of-merit and gives rise to negative refraction in the near- $\mathrm{IR}^{10}$, microwave $^{28}$ and optical regime ${ }^{13}$.

Manipulating sound waves is readily possible by utilizing metamaterials. Those materials are realized by resonating building blocks that are fabricated on a size scale smaller than the wavelength of the irradiated acoustic wave. It is the ability to control and tune those meta-atoms, which forms the basics in the design for tailored and unusual acoustic material responses. There are different strategies in creating negative refraction using acoustic metamaterials. One approach is to use perforations. An isotropic negative index can be obtained by coiling up small channels of perforations ${ }^{17}$. Hyperbolic metamaterial for broad-angle negative refraction on the other hand, can be constructed using layered holey structures ${ }^{18}$. Another approach is to employ locally resonating structures. The first metamaterial fabricated to possess double negativity (simultaneous negative effective bulk modulus $1 / \kappa$ and mass density $\rho$ ) was a one-dimensional tube design consisting of periodic interspaced open side branches and membranes ${ }^{29}$. Both of those resonators describe a Drude-like behavior for the effective bulk modulus and mass density respectively.

\section{Results}

Designer acousto-elastic surface modes. To go beyond the lumped element design we introduce structured metallic components which are able to convey tunable double negativity behavior in a planar configuration although extensions into 3 dimensions should not add any difficulties. For the design of such a material we take a close look at the EM fishnet metamaterial and initially aim at converting its plasmonic interpretation into acousto-elasticity ${ }^{13}$. In its basic design we have two adjacent holey films made out of good conductors ( $\mathrm{Au}$, $\mathrm{Ag}$ ) which are separated by a dielectric spacer. Metal surfaces punctured with subwavelength holes allow for the EM waves to penetrate much in the same way as a plasmon polariton ${ }^{30}$. Interestingly does the effective permittivity take the same plasma form whether they are supported in metals such as silver or in perforated highly conducting surfaces. It is this behavior which describes the electric response of a fishnet structure that is generated in the holey films. We will start out examining the acoustic analogy of these designer surface modes and 


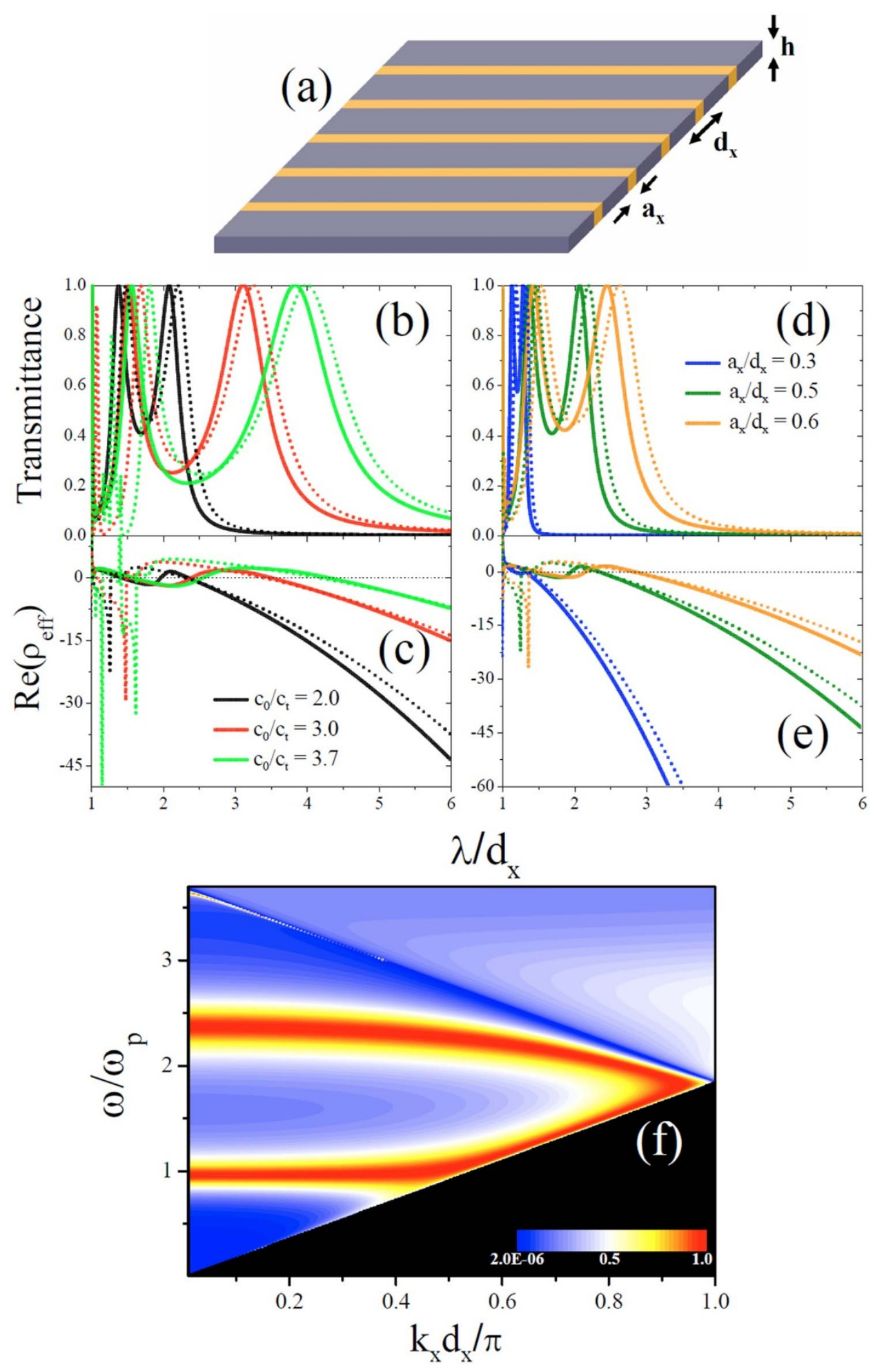

Figure 1 Transmittance and effective mass density spectra of the structured and elastically filled rigid screen. For all simulations, the normalized screen thickness is fixed at $h / d_{x}=0.7$ and the filling material has $\rho=2300 \mathrm{~kg} / \mathrm{m}^{3}$ and a longitudinal speed of sound $c_{l}=1410 \mathrm{~m} / \mathrm{s}$. Furthermore we consider the sample to be immersed in water, $c_{0}=1481 \mathrm{~m} / \mathrm{s}$ and $\rho_{0}=1000 \mathrm{~kg} / \mathrm{m}^{3}$. The transmittance $(\mathrm{b})$ and effective mass density $\operatorname{Re}\left(\rho_{\text {eff }}\right)(\mathrm{c})$ spectra are calculated for $a_{x} / d_{x}=0.5$. In (d,e) we hold $c_{0} / c_{t}=2$. The full (dotted) lines represent data obtained by modal expansions (COMSOL simulation). The incident sound plane wave is impinging at the normal direction. (f) The band diagram is simulated for a structure with the parameters $a_{x} / d_{x}=0.5$ and $h / d_{x}$ $=0.7$ and contains filling parameters as in the latter example though with $c_{0} / c_{t}=3.7$.

later study the effective bulk modulus which is showing resemblance to the magnetic response of the fishnet structure.

Let us suppose that we have a perfect rigid screen into which holes are carved. In order for an incident airborne sound field to be highly localized, we fill these indentations with an elastic material as rendered in Fig. 1a. We begin with a simple model that is constructed for a structure with translational invariance in the $y$ direction and textured by periodic slits of lattice constant $d_{x}$ along the $x$ axis. An 
incident wave excites elastic cavity modes inside the filled indentations which predominantly are governed by a displacement $u_{z}$ in the direction perpendicular to the lattice. The displacement generally satisfies the homogeneous wave equation for an isotropic solid:

$$
\rho \frac{\partial^{2} u_{z}}{\partial t^{2}}=\mu\left(\frac{\partial^{2} u_{z}}{\partial z^{2}}+\frac{\partial^{2} u_{z}}{\partial x^{2}}\right)+(\lambda+\mu)\left(\frac{\partial^{2} u_{z}}{\partial z^{2}}+\frac{\partial^{2} u_{x}}{\partial x \partial z}\right),
$$

where $\lambda, \mu$ and $\rho$ are the modulus of incompressibility (first Lamé coefficient), modulus of rigidity (second Lamé coefficient) and the solid mass density respectively. As the elastic inclusions are clamped to the rigid frame, $u_{z}\left(x=0, a_{x}\right)=0$ must vanish at the edges of the slit. Furthermore, we must guarantee zero shear-stress at the freespace and metamaterial interface as waves only propagate longitudinally in a fluid, $\sigma_{x z}=0$. Given the long wavelength limit which we are mainly interested in, i.e., $\lambda \gg a_{x}$, we take the fundamental cavity eigenmode to be dominant as it penetrates the deepest into the elastic inclusion, hence, we can write the out-of-plane displacement field inside this region as:

$$
u_{z}(x, z)=A \sin \left(\beta_{z} z\right) \cdot \sin \left(\frac{\pi}{a_{x}} x\right) \cdot e^{i w t} .
$$

Disregarding in-plane displacement $\left(u_{x}=0\right)$ is a plausible assumption in narrow elastic channels, hence we substitute Eq. (2) into Eq. (1), whereupon we solve for the wavenumber $\beta_{z}$ comprising longitudinal and transverse wave motion:

$$
\beta_{z}=\omega \sqrt{\frac{\rho}{\lambda+2 \mu}} \sqrt{1-\frac{\mu}{\rho} \frac{\pi^{2}}{a_{x}^{2}} \frac{1}{\omega^{2}}}=\frac{\omega}{c_{l}} \sqrt{1-\frac{\omega_{p}^{2}}{\omega^{2}}},
$$

which possesses a cut-off frequency $\omega_{p}=c_{t} \frac{\pi}{a_{x}}$. As we are focusing on the long wavelength regime we take into consideration only specular reflection when imposing continuity of an normally incident plane wave irradiating onto the structured half-space $(h \rightarrow \infty)$. From here it is straightforward to write down expressions for the reflection coefficient and the effective impedance:

$$
R_{0}=\frac{Z_{e f f}-1}{Z_{e f f}+1}, \quad Z_{\text {eff }}=\rho_{\text {eff }} c_{e f f}=\frac{\beta_{z}(\lambda+2 \mu)}{S_{f} \omega},
$$

where the structure factor $S_{f}=\frac{8}{\pi^{2}} \frac{a_{x}}{d_{x}}$. Substituting Eq. (3) into Eq. (4) and rewriting the effective speed of sound as $c_{\text {eff }}=k_{0} / \beta_{z}$, we arrive at:

$$
\rho_{\text {eff }}=\frac{\pi^{2} d_{x} \rho c_{0}}{8 a_{x}}\left(1-\frac{\omega_{p}^{2}}{\omega^{2}}\right),
$$

when solving for $\rho_{\text {eff }}$ which is the canonical Drude form of an acousto-elastic surface mode with a geometrical "plasma" frequency of

$$
\omega_{p}=c_{t} \frac{\pi}{a_{x}}
$$

the cut-off frequency of the elastic waveguide. In summary we can say that structured rigid screens will have this form of the effective mass density provided all dimensions are treated in the effective medium limit. The acousto-elastic cut-off frequency can be modified at will such that the screen can constitute a tunable high-pass filter with a stopband from zero frequency up the cut-off.

In the following we will take a look at the tunability of the structured screen with the aim to design the spectral location of the acoustic "plasma" frequency. In order to do so, we employ a rigid structured screen of finite thickness $h$ as depicted in Fig. 1a and calculate complex transmission and reflection coefficients for using them to numerically obtain the effective constitutive parameters based on a retrieval technique ${ }^{31}$. The theoretical formalism used to calculate the scattering coefficients is based on the modal expansion of the pressure and velocity fields in free-space such as the displacement and stresses inside the elastic inclusion ${ }^{32}$. Even though, in the former, the metamaterial halfspace is treated in the effective medium limit $\left(\lambda \gg a_{x}\right)$ by solely introducing specular reflections and taking into account only the fundamental elastic cavity mode, we conduct simulations by considering all higher expansion orders. As given in the caption of Fig. 1, we hold the screen thickness $h / d_{x}$ that is normalized to the lattice $d_{x}$ fixed, but vary the width $a_{x} / d_{x}$ of the indentations and also the inclusion material by different values of the transversal speed of sound $c_{t}$. Transmittance spectra are plotted and compared to the real part of the effective mass density $\operatorname{Re}\left(\rho_{\text {eff }}\right)$ as depicted in Fig. $1 \mathrm{~b}$ and Fig. $1 \mathrm{c}$ respectively. We clearly see that by lowering $c_{t}$ we also lower the onset of the first allowed band which means that the spectral locations of the acousto-elastic plasma frequencies shift towards longer wavelengths $\lambda / d x$. These are the locations at $\lambda_{p}=\frac{2 \pi c_{0}}{\omega_{p}}=2 a_{x} \frac{c_{0}}{c_{t}}$ where the effective mass density, as seen in Fig. $1 c$, changes sign - the transition between an opaque and transparent effective fluid. Fig. 1d and Fig. 1e confirm that the acoustoelastic plasma oscillation can be further controlled by the indentation width $a_{x}$. With a specific chosen filling material, in this case $c_{0} / c_{t}=$ 2.0 , we predict that the cut-off wavelengths now locate at $\lambda_{p}=4 a_{x}$. In order to verify the analytical approach based on modal expansions (full lines in Fig. 1), we have further conducted full-wave simulations (COMSOL Multiphysics, dotted lines) and found very good agreement. In addition we calculate the radiative transmittance band diagram which has been plotted in units of the plasma frequency. This is rendered in Fig. If (see the captions for details) where the low frequency plasmon-like mode interestingly shows very little dispersion with the parallel momentum $k_{x}$ of the incident sound. Again it must be noted that the cut-off can be controlled at will by the size of the indentations and the choice of the filling material such as polymers. This allows for the creation of designer surface states at almost arbitrary frequency.

Tunable negative effective bulk modulus. The magnetic response of the aforesaid EM fishnet structure is induced by the component consisting of metal-insulator-metal (MIM) units. These structures are hosting resonances which can give rise to negative values of the effective permeability. Recently a theoretical study on the acoustical version of the MIM structure showed that these sandwich-materials would give rise to an effective negative bulk modulus $1 / \kappa$, which sustains for a broad range of angles of incident sound ${ }^{33}$. The consequence of a negative bulk modulus in a fluid element is the overall expansion of it as a reaction to a positive acoustic stimulus. This behavior has recently been observed experimentally in the audio-frequency range ${ }^{34}$. In Fig. 2a we show the slitted version of this design and compute the normally incident sound transmittance through it by the same method as reported $\mathrm{in}^{33}$. We fill a fluid in the intermediate gap region of the structure (with height $h_{g}$ ) and study the controllability of the resonance by changing the value of the speed of sound $c_{g}$. We compare the spectral transmittance (Fig. 2b) to the retrieved effective bulk modulus $\operatorname{Re}(1 / \kappa)$ (Fig. $2 c)$ that at resonance sustain negative values over extended ranges. These regions of negative $1 / \kappa$ are as expected accompanied by polaritoniclike bandgaps, which in Fig. $2 b$ are seen by the presence of sharp dips in the transmittance spectra. Moreover, we predict a distinct correlation between these gap induced resonances and the speed of sound $c_{g}$ therein as the resonating modes shift towards lower frequencies when the filling material speed-of-sound is lowered.

Composite material exhibiting double negativity. The aforementioned tuning strategy will prove to be very useful at the stage when merging the previous Drude-like metamaterial containing negative $\rho_{\text {eff }}$ together with the present one to form a composite demonstrating 


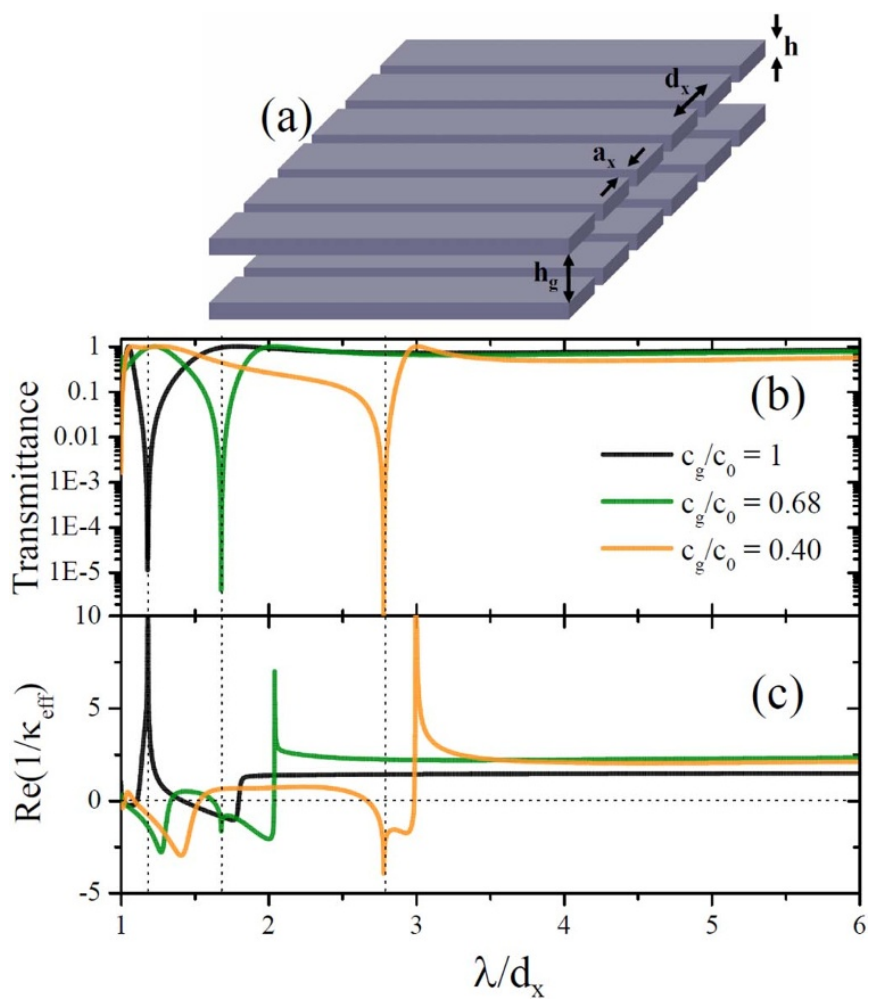

Figure $2 \mid$ Transmittance and effective bulk modulus $\operatorname{Re}(1 / \kappa)$ spectra for two facing structured rigid plates with $a / d_{x}=0.5, h / d_{x}=0.2$ and the gap separation $\boldsymbol{h}_{\boldsymbol{g}} / \boldsymbol{d}_{\boldsymbol{x}}=\mathbf{0 . 1}$. The gap is filled with a fluid of relative mass density $\rho_{g} / \rho_{0}=1$ and speeds of sound $c_{g} / c_{0}$ as indicated. The transmittance is presented in logarithmic scale and calculated at normal incidence. double negativity. The pursuit is to shift these bulk modulus $1 / \kappa$ resonances shown in Fig. 2, below the cut-off frequency $\omega_{p}$ of the elastomer filled screen. This particular design idea is a utile extension of the former one, we solely need to fill the slits with an elastic material as sketched in Fig. 3a. We design a plasma frequency to be located at $\lambda_{p} \approx 2.8 d_{x}$ below which the effective mass density is negative. We have chosen to use a fluid inside the gap of speed $c_{g} / c_{0}=$ 0.25 such that the negative response of $1 / \kappa$ occurs within the forbidden region, below the plasma frequency. The combination of simultaneous negative constitutive parameters induces a passband of full sound transmission at around $\lambda \approx 4.4 d_{x}$, as seen in Fig. $3 \mathrm{~b}$. Here again we compare analytical predictions with full-wave simulations where a fairly good agreement is obtained, confirming propagation at a region of double negativity. Interestingly we observe negative dispersion in this propagation band which has been computed by the transmittance as a function of frequency and parallel momentum, as rendered in Fig. 3c.

\section{Discussion}

We have demonstrated both by analytical and numerical simulations that structured rigid screens filled with elastic inclusions can be described in the long wavelength limit as an effective medium characterized by a mass density of the plasmon form. This type of metamaterial is thus not only used to control surface waves but also to create them. The combination of this behavior together with gap materials hosting bulk modulus resonances, produces negative dispersion with simultaneous negative values of $\rho_{\text {eff }}$ and $1 / \kappa_{\text {eff }}$, which is a characteristic of negative refractive index materials. The near-field coupling of the constituting resonances can be considered weak, which is essential when tuning the two effective parameters independently.
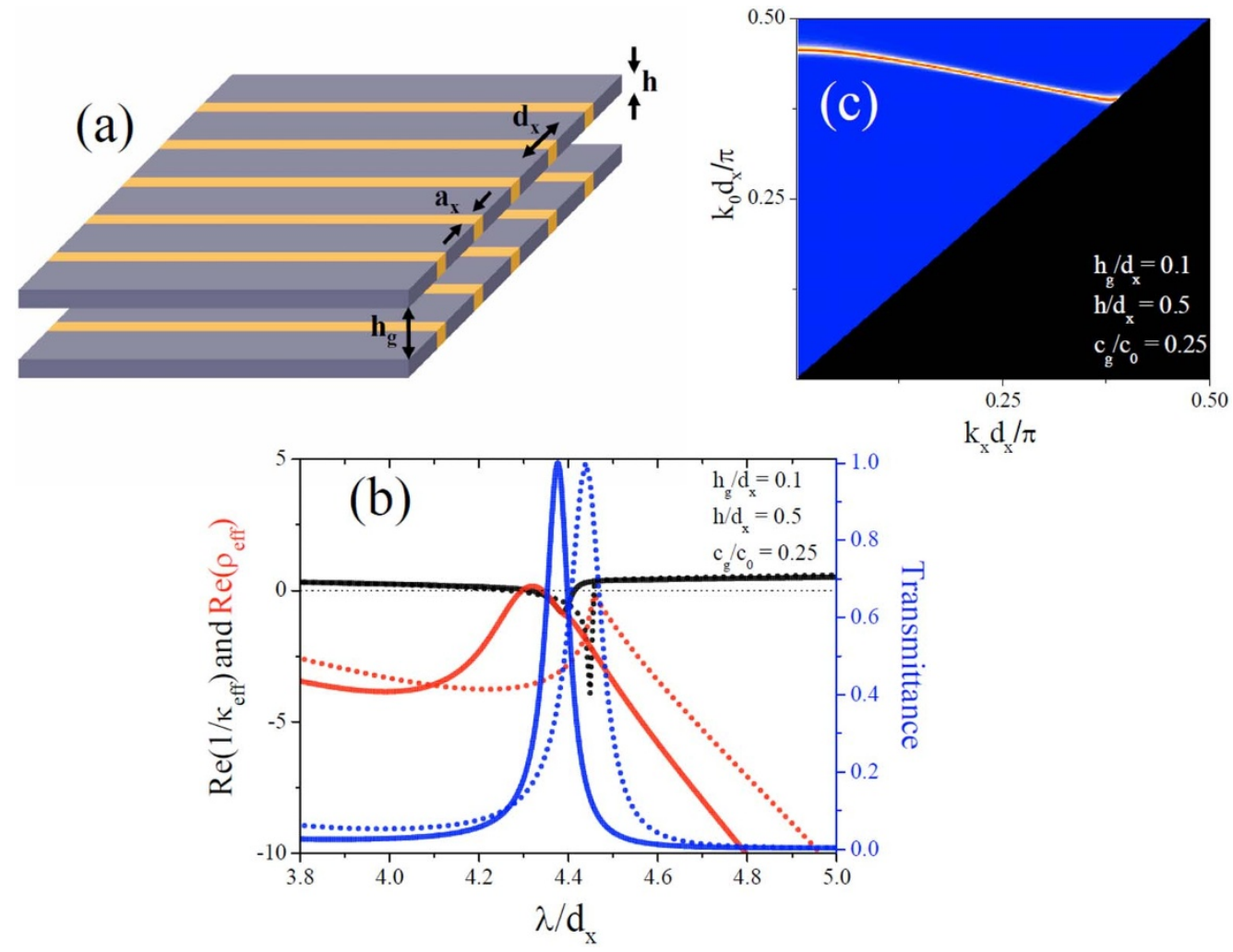

Figure $3 \mid$ Acoustic double negativity band in a composite metamaterial. (a) The composite metamaterial is designed with parameters $a / d_{x}=0.5, h / d_{x}=$ 0.5 and $h_{g} / d_{x}=0.1$ with an elastic inclusion containing $\rho=2300 \mathrm{~kg} / \mathrm{m}^{3}, c_{l}=1400 \mathrm{~m} / \mathrm{s}$ and $c_{t}=530 \mathrm{~m} / \mathrm{s}$. (b) With $c_{g} / c_{0}=0.25$ the transmittance spectra (right axis) is accompanied with plots of $\operatorname{Re}\left(1 / \kappa_{\text {eff }}\right)$ and $\operatorname{Re}\left(\rho_{\text {eff }}\right)$ (left axis). The full (dotted) lines represents data obtained by modal expansions (COMSOL simulation). (c) Band diagram simulation for the structure with parameters as given in (b). 


\section{Methods}

We have used modal expansion techniques (rigorously coupled modes theory) for analytically deriving the reflection coefficient and the impedance leading to the expression of the effective mass density in perforated elastically filled screens, Eq. (5). In the second stage, this technique has been implemented to numerically compute the transmission data both as a function of frequency $\omega$ and parallel momentum $k_{x}^{32,33}$.

Based on an effective medium retrieval technique, we were able to calculate the effective acoustic paramaters such as the mass density $\rho_{\text {eff }}$ and the bulk modulus $1 / \kappa_{\text {eff }}$. We have employed the method designed by D.R. Smith et al. and slightly modified it for the case of sound ${ }^{25}$.

The finite element method program COMSOL Multiphysics was utilized to conduct verifying numerical experiments seen in Fig. 1b - Fig. 1e, such as Fig. 3 b.

1. Pendry, J. B., Schurig, D. \& Smith, D. R. Controlling electromagnetic fields. Science 312, 1780-1782 (2006).

2. Chen, H., Chan, C. T. \& Sheng, P. Transformation Optics and Metamaterials. Nature Mater 9, 387-396 (2010).

3. Chen, H. \& Chan, C. T. Acoustic cloaking and transformation acoustics. J. Phys. D 43, 113001-113015 (2010)

4. Milton, G. W., Briane, M. \& Willis, J. R. On cloaking for elasticity and physical equations with a transformation invariant form. New J. Phys. 8, 248-268 (2006).

5. Cummer, S. A. et al. Scattering theory derivation of a 3D acoustic cloaking shell. Phys. Rev. Lett. 100, 024301-024304 (2008).

6. Dupont, G., Farhat, M., Diatta, A., Guenneau, S. \& Enoch, S. Numerical analysis of three-dimensional acoustic cloaks and carpets. Wave Motion 48, 483-496 (2011).

7. Popa, B. I., Zigoneanu, L. \& Cummer, S. A. Experimental Acoustic Ground Cloak in Air. Phys. Rev. Lett. 106, 253901-253904 (2011).

8. Stenger, N., Wilhelm, M. \& Wegener, M. Experiments on elastic cloaking in thin plates. Phys. Rev. Lett. 108, 014301-014305 (2012).

9. Veselago, V. G. The electrodynamics of substances with simultaneously negative values of $\varepsilon$ and $\mu$. Sov. Phys. Usp. 10, 509-514 (1968).

10. Zhang, S. et al. Experimental Demonstration of Near-Infrared Negative-Index Metamaterials. Phys. Rev. Lett. 95, 137404-137407 (2005).

11. Hoffman, A. J. et al. Negative refraction in semiconductor metamaterials. Nat. Mater. 6, 946-950 (2007).

12. Yao, J. et al. Optical Negative Refraction in Bulk Metamaterials. Science 321, 930 (2008).

13. Valentine, J., Zhang, S., Zentgraf, T., Ulin-Avila, E., Genov, D. A., Bartal, G. \& Zhang, X. Three Dimensional Optical Metamaterial Exhibiting Negative Refractive Index. Nature (London) 455, 376-379 (2008).

14. Li, J. \& Chan, C. T. Double-Negative Acoustic Metamaterial. Phys. Rev. E 770, 055602-055605(R) (2004).

15. Farhat, M, Guenneau, S., Enoch, S., Movchan, A. B. \& Petursson, G. G. Focussing bending waves via negative refraction in perforated thin plates. Appl. Phys. Lett. 96, 081909-081911 (2010).

16. Fok, L. \& Zhang, X. Negative acoustic index metamaterial. Phys. Rev. B $\mathbf{8 6}$ 214304-214312 (2011)

17. Liang, Z. \& Li, J. Extreme Acoustic Metamaterial by Coiling Up Space. Phys. Rev. Lett. 108, 114301-114304 (2012).

18. Christensen, J. \& Garcia de Abajo, F. J. Anisotropic Metamaterials for Full Control of Acoustic Waves. Phys. Rev. Lett. 108, 124301-124305 (2012).

19. Pendry, J. B. Negative refraction makes a perfect lens. Phys. Rev. Lett. 85, 3966-3969 (2000)

20. Ebbesen, T. W., Lezec, H. J., Ghaemi, H. F., Thio, T. \& Wolff, P. A. Extraordinary optical transmission through sub-wavelength hole arrays. Nature 391, 667-669 (1998).

21. Ambati, M., Fang, N., Sun, C. \& Zhang, X. Surface resonant states and superlensing in acoustic metamaterials. Phys. Rev. B 75, 195447-195451 (2007).
22. Hou, B. et al. Tuning Fabry-Perot resonances via diffraction evanescent waves. Phys. Rev. B 76, 054303-054308 (2007).

23. Li, J., Fok, L., Yin, X., Bartal, G. \& Zhang, X. Experimental demonstration of an acoustic magnifying hyperlens. Nature Mater. 8, 931-934 (2009).

24. Zhu, J. et al. A holey-structured metamaterial for acoustic deep-subwavelength imaging. Nat. Phys. 7, 52-55 (2011)

25. Smith, D. R., Padilla, W. J., Vier, D. C., Nemat-Nasser, S. C. \& Schultz, S. Composite Medium with Simultaneously Negative Permeability and Permittivity. Phys. Rev. Lett. 84, 4184-4187 (2000).

26. Pendry, J. B., Holden, A. J., Robbins, D. J. \& Stewart, W. .J. Magnetism from conductors and enhanced nonlinear phenomena. IEEE Microwav. Theory and Tech. 47, 2075-2084 (1999).

27. Pendry, J. B., Holden, A. J., Stewart, W. J. \& Youngs, I. Extremely Low Frequency Plasmons in Metallic Mesostructures. Phys. Rev. Lett. 76, 4773-4776 (1996).

28. Navarro-Cia, M., Beruete, M., Sorolla, M. \& Campillo, I. Negative refraction in a prism made of stacked subwavelength hole arrays. Opt. Express 16, 560-566 (2008).

29. Lee, S. H., Park, C. M., Seo, Y. M., Wang, Z. G. \& Kim, C. K. Composite Acoustic Medium with Simultaneously Negative Density and Modulus. Phys. Rev. Lett. 104, 054301-054304 (2010).

30. Pendry, J. B., Martin-Moreno, L. \& Garcia-Vidal, F. J. Mimicking Surface Plasmons with Structured Surfaces. Science 305, 847-848 (2004).

31. Smith, D. R., Schultz, S., Markos, P. \& Soukoulis, C. M. Determination of effective permittivity and permeability of metamaterials from reflection and transmission coefficients. Phys. Rev. B 65, 195104-195107 (2002).

32. Christensen, J., Christensen, J., Martin-Moreno, L. \& Garcia-Vidal, F. J. Theory of Resonant Acoustic Transmission through Subwavelength Apertures. Phys. Rev. Lett. 101, 014301-014304 (2008).

33. Christensen, J., Martin-Moreno, L. \& Garcia-Vidal, F. J. All-angle blockage of sound by an acoustic double-fishnet metamaterial. Appl. Phys. Lett. 97, 134106-134108 (2010).

34. Bell, J. S. et al. Low acoustic transmittance through a holey structure. Phys. Rev. B 85, 214305-214310 (2012).

\section{Acknowledgments}

J. C. gratefully acknowledges financial support from the Carlsberg Foundation under Contract No. MetaSound 2011-01-0099. J. L. thanks for the support from City University of Hong Kong SRG grant number 7002598 and the GRO program of Samsung Advanced Institute of Technology. J. C. would like to thank Luis Martin-Moreno for stimulating discussions.

\section{Author contribution}

J.C. and M.W. developed the analytical method. J.C. conducted numerical simulations based on the analytical method. J. L. and Z. L. undertook full-wave simulations (FEM). J.C. wrote the article and M.W. and J. L. participated in the revision. All authors contributed to discussions. J. C. guided the research.

\section{Additional information}

Competing financial interests: The authors declare no competing financial interests.

License: This work is licensed under a Creative Commons

Attribution-NonCommercial-NoDerivs 3.0 Unported License. To view a copy of this license, visit http://creativecommons.org/licenses/by-nc-nd/3.0/

How to cite this article: Liang, Z., Willatzen, M., Li, J. \& Christensen, J. Tunable acoustic double negativity metamaterial. Sci. Rep. 2, 859; DOI:10.1038/srep00859 (2012). 